Radial and Nonradial Pulsations as Probes of Stellar Physics

ASP Conference Series, Vol. 259, 2002

C. Aerts, T.R. Bedding, \& J. Christensen-Dalsgaard, eds.

\title{
Resonant Excitation of Nonradial Modes in RR Lyrae Stars
}

\author{
R. Nowakowski \\ Copernicus Astronomical Center, Warsaw, Poland
}

\begin{abstract}
A nonlinear development of radial pulsation instability to a resonant excitation of nonradial modes is studied. The theory covers the cases of axisymmetric $(m=0)$ modes as well as $(m,-m)$ pairs. Adopting a simplified treatment of the mode coupling, it is found that multimode pulsation with constant amplitudes is a highly probable solution. An observable consequence of the $m=0$ mode excitation is randomness of pulsation amplitude. The case of an $\ell=1$ mode is the most important because of a small averaging effect and a large excitation probability. A significant amplitude and phase modulation is predicted in the case of excitation of an $\ell=1, m= \pm 1$ pair. This may explain Blazhko-type modulation in RR Lyrae stars. If this model is correct, the modulation period is determined by the rotation rate and the Brunt-Väisälä frequency in the deepest part of the radiative envelope.
\end{abstract}

\section{Introduction}

One of the most intriguing questions concerning RR Lyrae stars is the nature of the long-term modulations that characterize about $20-30 \%$ of RRab and $2-3 \%$ of RRc stars (e.g., Moskalik \& Poretti, these proceedings; Kovács, 2000). The modulations of the oscillation amplitudes and phases manifest themselves in the Fourier spectra as secondary peaks with frequencies close to the main pulsation frequency. There seems to exist two distinguishable cases: one secondary peak with frequency either higher or lower than the main frequency and a pair of side peaks which, together with the main peak, form an equidistant triplet.

There are two models proposed to explain the modulations: a magnetic oblique rotator (Shibahashi, 2000) and the 1:1 resonance model (Van Hoolst et al., 1998). In this paper we develop the resonant model.

\section{Nonresonant properties of oscillation modes}

As was shown by Van Hoolst et al. (1998) and Dziembowski \& Cassisi (1999), there always exists a very dense spectrum of low- $\ell$ nonradial modes in the frequency range of the lowest radial overtones in models of RR Lyrae stars. The most unstable modes have frequencies very close to the fundamental as well as the first overtone frequencies. Among the low degree modes in the closest vicinity of the radial modes, the $\ell=1$ modes are the most unstable and thus the most important dynamically. 
Small frequency differences between the radial and the closest nonradial mode suggest that 1:1 and similar resonances should be taken into account in the study of the oscillations. Moreover, this frequency difference, which is an important parameter in resonant dynamics, is such a sensitive function of the stellar parameters that it is justified to treat it as a random quantity.

In the simplest nonlinear theory of stellar pulsations without resonances, sufficiently high amplitude of one mode may stabilize all other linearly unstable modes so that they are not present in oscillations. This simple saturation mechanism leads to monomode pulsations which are typical for RR Lyrae stars.

\section{Resonant coupling and instability of monomode pulsations}

One of the conditions for the resonant coupling between different modes may be written as $\sum_{i} m_{i}=0$, where $m_{i}$ are azimuthal numbers of the interacting modes. For the most important case of $\ell=1$ it means two possibilities:

- coupling of the radial mode with an $m=0$ nonradial mode,

- coupling of the radial mode with an $m=1, m=-1$ pair.

It turns out that the two cases are dynamically equivalent, i.e., the amplitude equations describing the mode amplitudes are in both cases the same with the same coefficients and in the latter case $A_{1,1}=A_{1,-1}$.

Radial monomode pulsations become resonantly unstable when

$$
|\Delta \omega|<\sqrt{|R|\left|A_{0,0}\right|^{2}-\kappa_{l, N}^{2}}
$$

(Van Hoolst et al., 1998), where $\kappa_{l, N}$ is a nonlinear growth rate of the nonradial mode, $R$ is a resonant coupling coefficient, $A_{0,0}$ is a radial mode amplitude, and the detuning parameter is given by

$$
\Delta \omega= \begin{cases}\omega_{1,0}-\omega_{0,0} & \text { for } m=0 \\ \left(\omega_{1,1}+\omega_{1,-1}\right) / 2-\omega_{0,0} & \text { for } m= \pm 1 \text { pair. }\end{cases}
$$

\section{Finite amplitude development of resonant instability}

In order to simplify the problem we made some assumptions. The most important are the use of adiabatic resonant coupling coefficients and the neglect of nonresonant nonlinear frequency changes. The former assumption was used by Van Hoolst et al. (1998) and Dziembowski \& Cassisi (1999). The latter assumption seems to be justified because the nonlinear frequency change has the same sign for both modes and it does not change the frequency difference significantly.

We first studied double-mode stationary solutions of amplitude equations together with their stability with respect to small perturbations. The parameters of the equations were calculated for the range of stellar models by Dziembowski \& Cassisi (1999). A typical solution is presented in Fig. 1. All interesting pairs of modes in all models give qualitatively the same solutions.

The $a$-branch solution exists if the detuning parameter is smaller than the critical value given by Eq. (1) (about 0.6 in the case presented in Fig. 1) and it is 


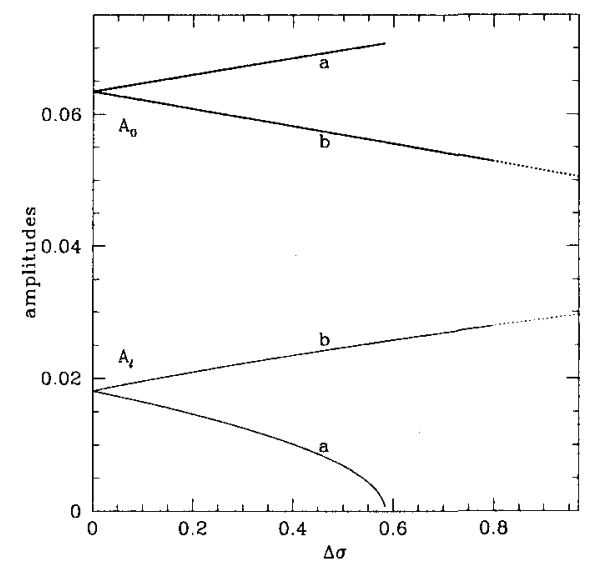

Figure 1. Double-mode stationary solutions for the radial fundamental mode (thick line) and the nearest $\ell=1, m=0$ nonradial mode (thin line) for a chosen RR Lyrae model. The dimensionless detuning parameter $\Delta \sigma$ is defined as $\Delta \omega / \kappa_{0,0}$. The problem is symmetric with respect to the change of the sign of the detuning parameter and the presented range of this parameter corresponds to the maximum value given by the density of the nonradial modes spectrum. Dotted lines indicate unstable solutions. For $\Delta \sigma$ smaller than approximately 0.6 there is a pair of solutions denoted by $a$ and $b$.

always stable. If the detuning parameter is higher than the previously mentioned critical value the monomode solution (not shown in Fig. 1) is stable, according to Eq. (1). This means that there always exists at least one stable fixed-point solution, either monomode (large $\Delta \omega$ ) or double-mode (small $\Delta \omega$ ).

Direct time integration of the amplitude equations shows that the timedependent solutions always converge to one of the stable fixed-point solutions.

Multimode fixed-point solutions of resonant amplitude equations are characterized by the phase-lock phenomenon (for the general discussion see Buchler et al., 1997). In our cases it means:

- for interaction with an $m=0$ nonradial mode, the two frequencies are equal,

- for interaction with an $m= \pm 1$ pair, the three frequencies are equidistant.

\section{Observational consequences}

When resonant interaction leads to excitation of an $m=0$ nonradial mode, an observer would see only one frequency due to the phase-lock phenomenon. The only effect of the presence of the nonradial mode is the aspect-dependence and thus the randomness of the observed pulsation amplitude. 
The excitation of the $m= \pm 1$ pair leads to the observed triplet-type modulation, where the side peaks in the spectrum are rotationally split $m= \pm 1$ modes. A simple estimate yields the splitting to be equal to $\bar{\Omega} / 2$, where $\bar{\Omega}$ is the mean rotation rate weighted with the Brunt-Väisälä frequency which is strongly peaked in the deepest part of the radiative envelope. Thus, in this model the Blazhko period is the measure of the rotation in this region.

\section{Conclusions and future work}

The resonant model presented in this paper predicts many observed features of RR Lyrae stars: random occurrence and ranges of the modulations as well as lower occurence rate of Blazhko stars among RRc than among RRab stars.

However, this model has some problems, too. The most important one is that it does not predict the strong asymmetry of the side-peak amplitudes (Kovács, these proceedings). Moreover, it does not explain features which have been recently observed: larger frequency splittings among $\mathrm{RRc}$ than among RRab stars (Moskalik \& Poretti, these proceedings) and the change of the Blazhko-period (Smith et al., these proceedings).

To understand the nature of the long-term modulations of RR Lyrae stars more efforts should be made both theoretically and observationally. The most promising are attempts to find the nonradial mode characteristics in line-profile variations of RR Lyrae itself (Kolenberg et al., these proceedings). Precise spectroscopic observations of other Blazhko stars would also be of great importance.

Our model also needs some improvements. The most important one is to take into account previously neglected coupling coefficients and more interacting modes, in particular the whole $\ell=1$ triplet. (2001)

This paper summarizes the results given by Nowakowski \& Dziembowski

Acknowledgments. We would like to thank the organizers for the financial support. We are also greatful to Wojtek Dziembowski and Geza Kovács for their useful suggestions given during the preparation of our talk and this paper.

\section{References}

Buchler, J.R., Goupil, M.-J., \& Hansen, C.J. 1997, A\&A, 321, 159

Dziembowski, W.A., Cassisi, S. 1999, Acta Astron., 49, 371

Kovács, G. 2000, in Nonlinear Studies of Stellar Pulsations, eds. M. Takeuti and D. Sasselov, (Kluwer Academic Publishers), 257, 61

Nowakowski, R.M. \& Dziembowski, W.A. 2001, Acta Astron., 51, 5

Shibahashi, H. 2000, in ASP Conf. Ser., Vol. 203, The Impact of Large Scale Surveys on Pulsating Star Research, eds. L. Szabados \& D. Kurtz, (San Francisco ASP), 299

Van Hoolst, T., Dziembowski, W.A., \& Kawaler, S.D. 1998, MNRAS, 297, 536. 